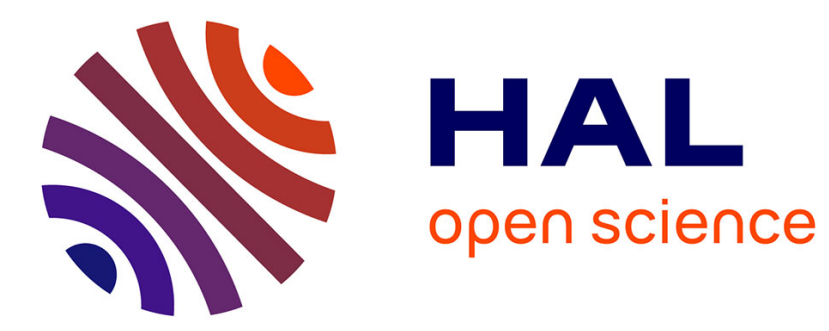

\title{
3D Haptic Handling of Microspheres
}

\author{
Aude Bolopion, Hui Xie, Sinan Haliyo, Stéphane Régnier
}

\section{To cite this version:}

Aude Bolopion, Hui Xie, Sinan Haliyo, Stéphane Régnier. 3D Haptic Handling of Microspheres. 2010 IEEE/RSJ International Conference on Intelligent Robots and Systems (IROS 2010), Oct 2010, Taipei, Taiwan. pp.6131-6136, 10.1109/IROS.2010.5650443 . hal-02912199

\section{HAL Id: hal-02912199 https://hal.science/hal-02912199}

Submitted on 5 Aug 2020

HAL is a multi-disciplinary open access archive for the deposit and dissemination of scientific research documents, whether they are published or not. The documents may come from teaching and research institutions in France or abroad, or from public or private research centers.
L'archive ouverte pluridisciplinaire HAL, est destinée au dépôt et à la diffusion de documents scientifiques de niveau recherche, publiés ou non, émanant des établissements d'enseignement et de recherche français ou étrangers, des laboratoires publics ou privés. 


\title{
3D Haptic Handling of Microspheres
}

\author{
Aude Bolopion, Hui Xie, Member, IEEE, D. Sinan Haliyo, Stéphane Régnier
}

\begin{abstract}
In this paper, a fully teleoperated 3D micro assembly task with haptic feedback is presented. Microspheres (diameter: $4-6 \mu \mathrm{m}$ ) are manipulated by pick-and-place. The setup is composed of a dual-tip gripper controlled through a haptic interface. To grasp the spheres, the tips must be correctly positioned with respect to the objects. The approach proposed to align the gripper is based on a user-driven exploration of the tobe-manipulated object. During this step, the haptic feedback is based on amplitude measurements from cantilevers in dynamic mode. Hence, the operator perceives the contact while freely exploring the manipulation area. A virtual guide is generated to pull the user to the optimum contact point, allowing correct positioning of dual tips. For the pick-and-place operation, the haptic feedback provides the user with information about the microscale interactions occurring during the operation. As experimental validation, a two-layer pyramid composed of four nylon microspheres is built in ambient conditions.
\end{abstract}

\section{INTRODUCTION}

Manipulation of objects of less than ten micrometers is a challenging issue as it shares many difficulties with nanomanipulation. Among them, the visual feedback under optical microscope is limited and do not enable the accurate positioning of tools and objects. Due to the scale reduction, adhesion forces become predominant over gravitational ones [1]. Tools to manipulate these objects must be carefully designed [2]. Fully automated micromanipulation is difficult to achieve, due to the high influence of environmental parameters, the lack of repetability and mainly the lack of intuitiveness of the overall system. Haptic feedback appears as a promising solution to provide assistance to the operators [3], in particular for AFM based manipulation [4], [5]. Solutions to assist micromanipulations through haptic feedback are first steps towards intuitive nanomanipulations.

Early examples of teleoperation with force feedback at this scale deal only with feeling the shape of a substrate or objects [6]. [7] reports a haptic implementation of a approach/retract task of an AFM probe. First remote tasks inducing a modification of the sample are indentations (e.g. direct patterning on a substrate [8]). Tasks involving pushing/pulling or cutting objects are also of primary interest [9], [10]. As only two measurements are directly available from an AFM cantilever (bending and torsion), 3D haptic feedback of nanoscale interactions between the tool and the object can only be achieved by the use of contact mechanics models. Such models are used for 3D haptic feedback in surface indentation and touching micro-objects [11], [12], [13]. However, so far, no manipulation tasks are reported.

The authors are with Institut des Systèmes Intelligents et de Robotique, Université Pierre et Marie Curie - Paris 6, CNRS UMR 7222, 4 Place Jussieu, 75005 Paris, France. \{bolopion, xie, haliyo, regnier\}@isir.upmc.fr
All the above mentioned works use a single AFM cantilever and static measurements. Consequently, only one controllable contact point is available to the user. Using the AFM in contact mode implies also some limitations compared to dynamic mode: the dynamic mode where the force measurement is obtained through variations on the amplitude or frequency of a vibrating probe is generally considered of finer quality than static contact mode, where the measured force is directly proportional to the deflection of the probe [14]. In addition, on the above mentioned works, a frequent time-consuming factor is the necessity of a preliminary scan, especially in the case where vision quality is poor (for objects smaller than few micrometers), or contact mechanics models are used for 3D feedback, or path planning is required to implement virtual guides.

We report here a complex fully teleoperated 3D microassembly task, consisting of building a two layer pyramidal structure from four $\varnothing 5 \pm 1 \mu \mathrm{m}$ microspheres. It is based on the analysis of haptic coupling schemes we did previously [15]. First results of 2D teleoperation of $\varnothing 50 \mu \mathrm{m}$ microspheres with haptic feedback using rolling were obtained in [16]. This current report deals with 3D microassembly in ambient conditions of microspheres ten times smaller. The system uses two independent AFM probes to collaboratively grasp and position each object, as reported in [17]. Teleoperation through haptic feedback is extensively used in every step of the operation and is based on the dynamic mode AFM. As the proposed approach does not aim for full automation but instead relies on the operator to increase the flexibility of the system, the pre-scan step is avoided and is replaced by a user guided initial exploration. This exploration allows for an in-line calculation of virtual guides, helping the operator to correctly align the dual tip gripper with respect to the manipulated object, even in the case of poor visual accuracy. Additionally, a haptic feedback scheme is presented for pick-and-place of microspheres.

This paper is organized as follows. The experimental setup and the manipulation protocol are described in Section II and Section III respectively. Haptic feedback based on dynamic mode measurements and in-line construction of virtual guides to accurately align the grippers to microspheres are discussed in Section IV. Section V depicts pick-and-place experiments and their detailed analysis.

\section{EXPERIMENTAL SETUP}

Detailed specifications of the manipulation setup are discussed in [17]. A brief summary is given here. The micromanipulation platform is depicted in Fig. 1. The AFM 


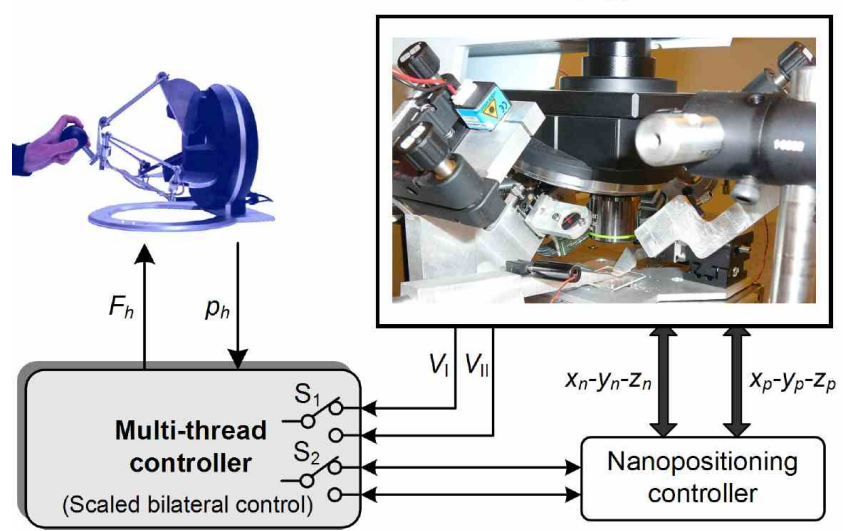

Fig. 1. AFM gripper based telemicromanipulation system. (left) The haptic device providing a user interface to control the three-dimensional microassembly with real time haptic feedback. (right) The dual-probe gripper comprised of two AFM cantilevers with protruding tips for pickand-place micromanipulation.

gripper is equipped with an optical microscope, and two sets of nanopositioning devices and optical levers to coordinate two AFM cantilevers with protruding tips (namely, Tip I and Tip II, Nanosensors ATEC-FM) facing each other, forming a dual-tip gripper. Tip I is fixed on a $X-Y-Z$ motorized micropositioning stage (resolution: $50 \mathrm{~nm}$ ), while Tip II is actuated by an open-loop $X-Y-Z$ piezotube (sub-nm resolution) mounted on a $X-Y-Z$ manual stage (resolution: 0.5 $\mu \mathrm{m})$. A closed-loop $X-Y-Z$ nanostage (resolution: $0.1 \mathrm{~nm}$ ) is used to actuate the sample holder during microassembly. Coarse alignment of the Tip I and Tip II is achieved under the optical microscope with large displacements of motorized and manual stages.

Each cantilever disposes of its own optical lever, comprised of a laser source and a four-quadrant photodiode. Data acquisition occurs at 500-800 $\mathrm{Hz}$ for static force sampling and at $600 \mathrm{kHz}$ for amplitude through a NI 6289 ADC.

Cantilevers can be used in two different modes: tapping and static. For the tapping mode, a piezoceramic excites each probe at its natural frequency. The amplitude of resulting oscillations is measured through the variations of the voltage output on the photodiode:

$$
A=\beta \cdot \Delta V
$$

where $A$ is the amplitude measurement, $\beta=10^{-6} \mathrm{~m} \cdot \mathrm{V}^{-1}$ is a calibrated conversion factor and $\Delta V$ is the differential voltage response of the photodiode.

In static mode, the normal force applied on the cantilever $F$ is measured directly from the output voltage of the photodiode:

$$
F=k_{n} S_{n} \Delta V
$$

where $k_{n}=2.8 \mathrm{~N} \cdot \mathrm{m}^{-1}$ is the normal stiffness of the cantilever, and $S_{n}=8 \cdot 10^{-7} \mathrm{~m} \cdot \mathrm{V}^{-1}$ is the sensitivity of the optical levers.
An Omega haptic interface, manufactured by Force Dimension ${ }^{1}$ is provided for intuitive user control of the manipulator. This master arm is a 3 degrees of freedom device. The user manipulates the handle and the resulting position $p_{h}$ is scaled down to be used to control the actuators (nanostage and piezotube). The haptic force $F_{h}$ sent to the user through the haptic interface is based on measurements from the two photodiodes $\left(V_{I}\right.$ and $\left.V_{I I}\right)$. As represented by the switches $S_{1}$ and $S_{2}$ in Fig. 1, different translators and feedbacks are used at each step of the microassembly. Next sections detail the use of the haptic interface to interactivly perform a microassembly task.

\section{3D Microassembly Protocol}

The manipulation area and a coarse positioning of the tips is determined using the optical microscope. The operator then places sequentially each tip on both sides of the object using the Omega, controlling respectively the sample holder through the nanostage and Tip II through the piezotube. In both steps the haptic feedback is provided based on the amplitude variations of each tip in tapping mode. Once the object is hold between two tips, the lift-off and release are achieved by haptic control of the sample holder. Contact mode measurement are used to provide the force feedback.

The approach proposed here is based on a user-driven exploration of the manipulated object. The haptic feedback allows the operator to feel when he/she touches the object while freely exploring the manipulation area. Note that during this operation the vertical position of the probes are constrained to a few micrometers above the substrate and the operator controls only the horizontal motion. The data recorded during this exploration is processed in-line and generates a virtual guide to pull the user to the optimum contact point. User "feels" and sequentially adjusts the contact force for both tips, assuring an adequate grip on the object. In the third phase of the manipulation, both grippers are immobilized on both sides of the object and the operator controls the motion of the sample holder (this method ensures that the displacement of both of the tips with respect to the substrate is identical), while still receiving haptic feedback calculated from the output of two probes.

Tip-alignment phases, including the haptic feedback and virtual guide generation and pick-and-place phases with force feedback are detailed in the following.

\section{Assisted Gripper Alignment}

The alignment of each tip is a user-driven process. The operator moves the tip while receiving haptic feedback derived from amplitude measurements of the AFM probe. During the initial exploration and prior to the generation of virtual guides, the haptic feedback is only on the $x$ axis (Fig. 2(a)). As the operator scans manually the surface of the tobe-manipulated object, the data is recorded to reconstruct its shape and create the virtual guide. This virtual guide generates the haptic feedback along $y$ axis, pulling the tip

\footnotetext{
${ }^{1}$ http://www.forcedimension.com
} 
to the calculated grasp line $y_{0}$, parallel to the $x$ axis and crossing the sphere's center.

\section{A. Tapping Mode Measurements}

In tapping mode, each probe is excited at its natural frequency. At constant height from the substrate and away from objects, this results in oscillations on a constant amplitude, noted $A_{0}$. While approaching an object, starting from a few hundreds of nanometers, the tip contacts the object intermittently and the amplitude $A_{t}$ decreases until a minimum value $A_{C P}$ is reached at full-contact between the tip and the object.

Fig. 2 illustrates the principle of object detection from amplitude variations. The tip is first set to a given height from the substrate $h_{0}$. This step is achieved in an initial phase and the user controls the motion only in the $(x, y)$ plane parallel to the substrate. While the tip moves on the grasp direction of the gripper, the $x$ axis, amplitude decreases until contact (Fig. 2(b)). On the $y$ axis, perpendicularly to the grasp direction, both tips must be aligned with the center of the sphere. This matches the minimum of amplitude along the $y$ axis, at a fixed $x$ position (Fig. 2(c)).

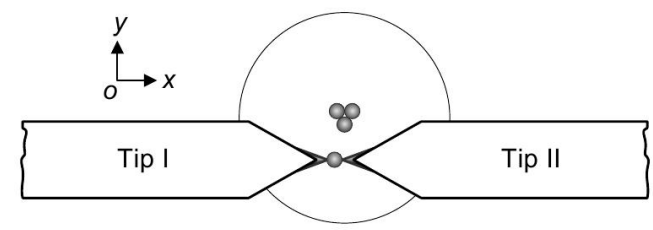

(a)

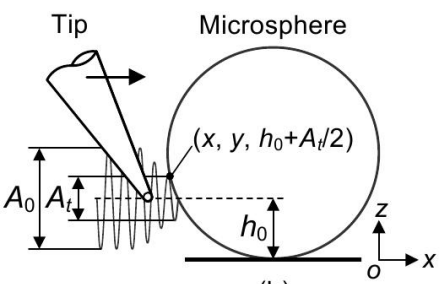

(b)

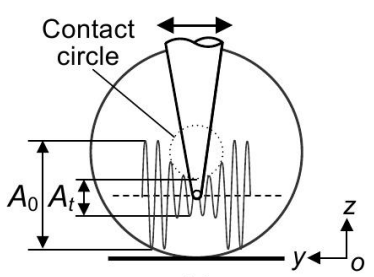

(c)
Fig. 2. A schematic diagram of haptic exploration by local scan of the lower semi-microsphere using a oscillating cantilever. (a) Top view of desired grasp configuration (b) Front view shows the tip tapping the microsphere while approaching on $x$-axis. (c) Side view shows the tip tapping the microsphere when scanning on $y$-axis with a fixed $x$ position.

\section{B. Haptic Feedback for Tip Alignment}

To align the gripper with the sphere and bring it to contact the visual feedback from the optical microscope does not provide sufficient resolution. Haptic feedback aims to compensate for this lack of visual feedback. The haptic coupling used is depicted in Fig. 3. Each tip is sequentially aligned on the grasp line and brought to contact.

1) $x$ axis: The haptic feedback along the $x$ axis should provide following information:

- $R_{1}$ : force null when the tip is far from the object

- $R_{2}$ : increasing force as the tip approaches the object

- $R_{3}$ : increasing force as the tip applies a force on the object

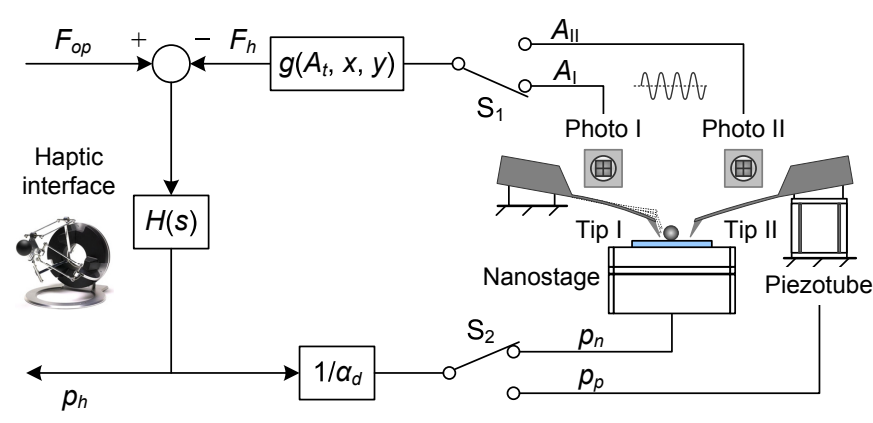

Fig. 3. Haptic coupling for dual tip gripper alignment. The user manipulates the actuators by setting the position of the haptic device. Haptic feedback is derived from amplitude measurements. Depending on the considered tip, the switches $S_{1}$ and $S_{2}$ enable to manipulate the nanostage or the piezotube, and accordingly receive the amplitude measurement from the photodiode I or II.

According to the variation of amplitude described previously, the following haptic feedback $F_{h x}$ is proposed and satisfies requirements $R_{1}-R_{3}$ :

$$
F_{h x}= \begin{cases}-\alpha_{a}\left(A-A_{0}\right) & \text { if } A>A_{C P} \\ -\alpha_{a}\left(A-A_{0}\right)+k_{x}\left(x-x_{C P}\right) & \text { else }\end{cases}
$$

where $\alpha_{a}$ is a scaling factor. The amplitude $A_{0}$ is measured at the beginning of the experiment while the tip oscillates at its natural frequency. Two cases are distinguished:

- before contact (first equation): as the amplitude is decreasing while the tip approaches the object, an increasing repulsive force is sent to the user so that he or she is aware of the presence of the object

- while in contact (second equation): a spring $k_{x}$ between the position of the contact point $x_{C P}$ and the current position of the tip $x$ is added to the feedback of the first equation. It simulates the force applied by the tip to the sphere. The contact point location $x_{C P}$ is acquired and set when the amplitude measurement reaches $A_{C P}$.

2) $y$ axis: The force perceived along the $y$ axis must enable the user to align the tip with respect to the sphere, on the grasp line. The haptic feedback along the $y$ axis is not available before all the points have been recorded and the computation of virtual guide is achieved. During this exploration in search of the $y_{0}$ position, the $x$ axis haptic feedback is provided to the user so that he or she perceives the sphere's location.

When $y_{0}$ is computed, a haptic feedback $F_{h y}$ simulating a spring $k_{y}$ between $y_{0}$ and the current position $y$ of the tip is sent to the user:

$$
F_{h y}=k_{y}\left(y-y_{0}\right)
$$

\section{Virtual Guide Generation}

During the initial exploration in tapping mode, $n$ contact points $\left(x_{t}^{i}, y_{t}^{i}\right)$, with their matching amplitudes $A_{t}^{i}(i=1 . . n)$ are collected. The contact position data is acquired only if the actual amplitude $A_{t}$ is in $\left[15 \% A_{0}, 70 \% A_{0}\right]$ interval to avoid inaccurate measurements. In order to define the $z_{t}^{i}$ coordinate for each contact point $\left(x_{t}^{i}, y_{t}^{i}\right)$, the approximation $z_{t}^{i}=A_{t}^{i} / 2$ 
is proposed. This is a relative position since the cantilever is oscillating around the height $h_{0}$ set manually. The calculation of the $z_{0}$ coordinate of the sphere is thus relative to $h_{0}$, and is not accurately known. However, as the only parameter useful for haptic feedback is the $y_{0}$ coordinate, this approximation is acceptable.

$n$ points $\left(t_{1}, \ldots t_{n}\right)$ are recorded during the exploration process. These points are used to reconstruct the shape of the manipulated sphere, calculate the grasp line and provide the haptic feedback along $y$ axis. With prior knowledge of the shape of the object, and the $n$ recorded points, the sphere can be reconstructed from the surface equation:

$$
\left(x-x_{0}\right)^{2}+\left(y-y_{0}\right)^{2}+\left(z-z_{0}\right)^{2}=R^{2}
$$

where $R$ is the radius of the sphere, and $x_{0}, y_{0}$ and $z_{0}$ are the coordinates of its center.

A least mean square algorithm is used to compute the parameters of the sphere (center and radius) to best fit the $n$ recorded points. The position $y_{0}$ of the grasping point along the $y$ axis is then known. Note that as all the points are on the same side of the sphere along $x$ axis $(x<0$ for Tip I and inversely for Tip II), the calculated $x_{0}$ coordinate may be inaccurate. However, as stated above, the only parameter used for virtual guide is $y_{0}$.

\section{Experimental Validation of Tip Alignment}

Manipulated objects are nylon microspheres, with a diameter of $4-6 \mu \mathrm{m}$. Both tips are first positioned manually at the correct height (around 500-600nm) above the substrate, using dynamic mode detection of the sample. [15] proposes a haptic feedback solution for this step. Each tip is then sequentially positioned by the operator at each side of the object.

1) $x$ axis haptic heedback: Experimental results acquired while moving the tip along $x$ axis and contacting the microsphere are depicted in Fig. 4. The position of the tip is represented in Fig. 4(a), the amplitude measurement is depicted in Fig. 4(b) and the haptic feedback in Fig. 4(c). In area 1, the tip is away from the sphere and the feedback is null. In area $\mathbf{2}$, the user distinctly perceives the haptic feedback as the tip approaches the sphere and intermittent contact starts. An additional feedback is transmitted when an effort is applied by the cantilever on the sphere in area $\mathbf{3}$, increasing the sensation of stiffness.

Compared to using direct force measurement from a cantilever in static mode, tapping mode amplitude measurement enables a better sensitivity on $x$ axis. In static mode, as the measurement direction is almost aligned with the probes' length, the equivalent stiffness is extremely high compared to $k_{n}$ on $z$ axis. Hence, a static detection on $x$ axis would only occur when a quite important force is already applied on the object. On the contrary, the use of tapping mode allows an earlier detection of the object as only intermittent contact with the object produces a detectable signal. This allows the user to be aware of the object's presence and avoids to push it involuntarily.
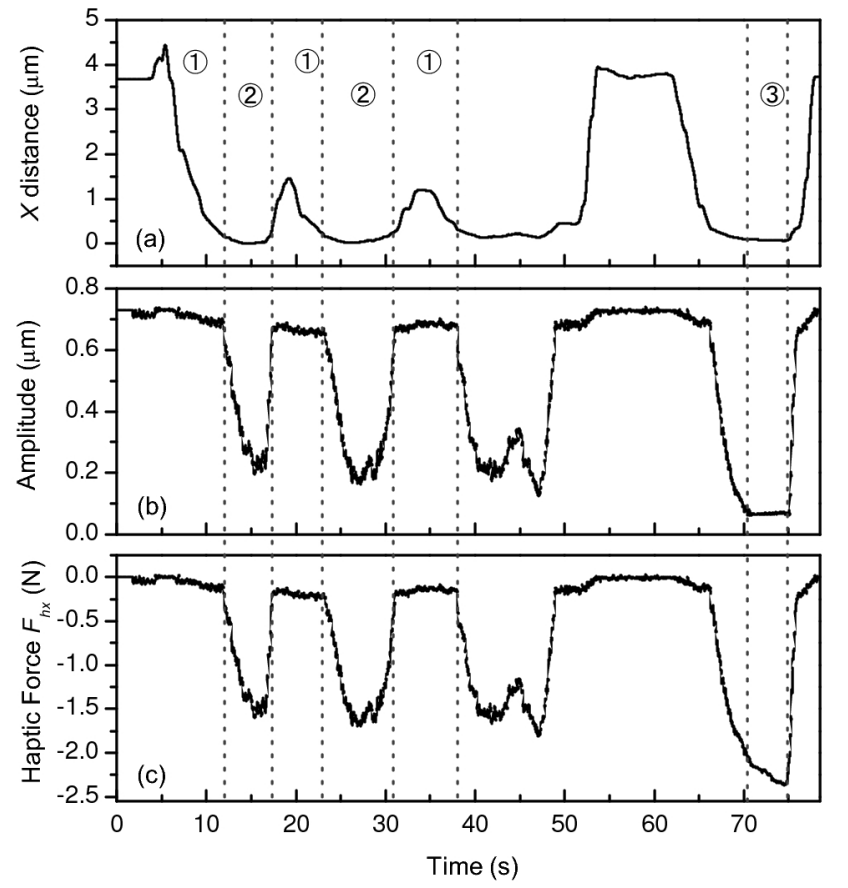

Fig. 4. Haptic feedback along the $x$ axis while exploring the to-bemanipulated sphere; (a) position of the tip, (b) amplitude measurement, and (c) force sent to the user. For the haptic feedback, the coefficients are set to: $\alpha_{a}=3 \cdot 10^{6} N . m^{-1}, k_{x}=10 \cdot 10^{6} N . m^{-1}$ and $a_{C P}=0.1 \mu \mathrm{m}$.

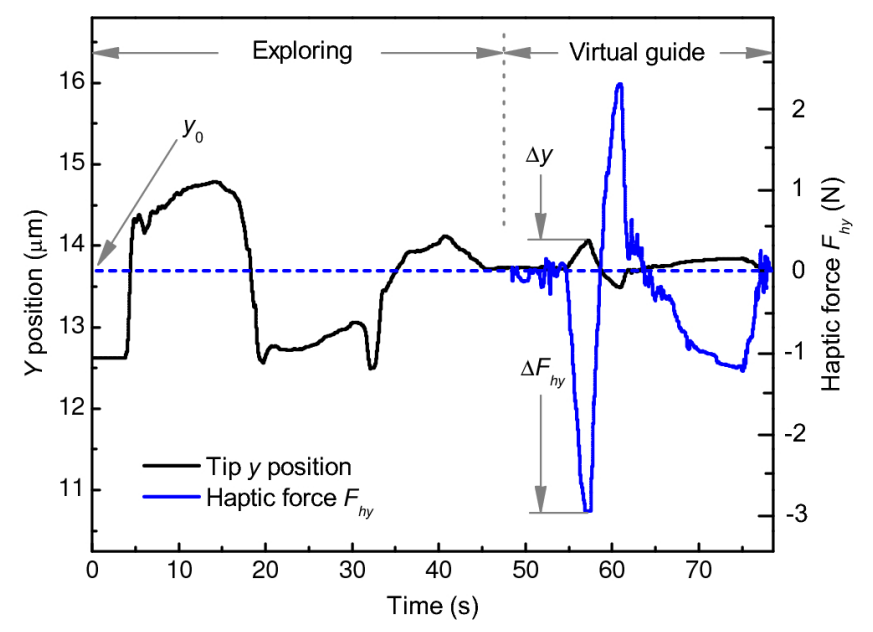

Fig. 5. Haptic feedback along the $y$ axis while aligning the tip with the sphere: position of the tip and force sent to the user. $k_{y}=10 \cdot 10^{6} \mathrm{~N} . \mathrm{m}^{-1}$

2) y axis haptic feedback: The accuracy of the virtual guide depends on the number of recorded points and their relative position. The influence of two parameters, the number of points $n$ and the minimum distance between two points (noted $d$ ) are empirically optimized. Used values are $n=12$ and $d=0.3 \mu \mathrm{m}$. $y$ axis feedback is effective as soon as the virtual guide is generated. Its value is calculated using Equation (2). Fig. 5 represents the force perceived by the user. The position of the tip as well as $y_{0}$ are also given.

The haptic feedback on the $y$ axis helps the user to align the tip with respect to the sphere as $y_{0}$ is at the equilibrium 
point of the virtual spring. Precise positioning is achieved since contact information is transmitted to the user through the $x$ axis of the haptic device $F_{h x}$, and alignment is ensured thanks to the haptic force $F_{h y}$.

\section{Pick-AND-Place With Haptic FeEdback}

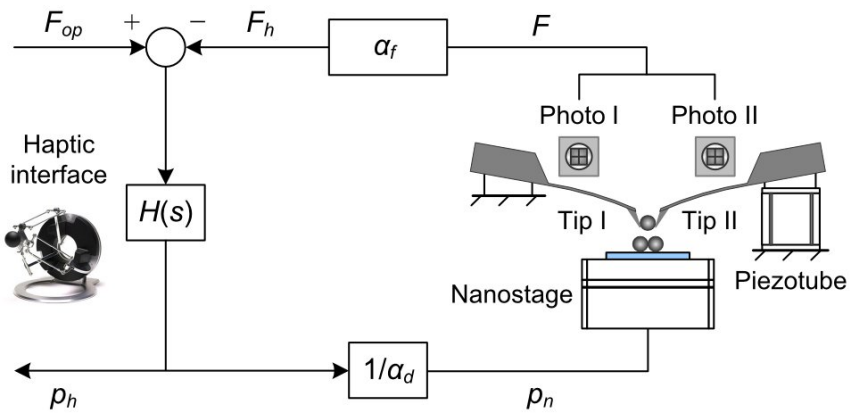

Fig. 6. Haptic coupling for pick-and-place. The user manipulates the nanostage with respect to the two tips by setting the position of the haptic device. Haptic feedback is derived from force measurement from the sum of the two photodiodes' outputs.

After the object is grasped between both tips, it will be lifted from substrate, transported to the target location and released. Haptic feedback is proposed for this task to render to the user the forces measured by both probes, with proper scaling. As depicted in Fig. 6 the Omega haptic device is used for position control of the sample holder through the nanostage, while the 2 tips holding the microsphere are immobilized. The force data is obtained in static mode, from the deflection of each probe as measured directly on photodiodes using Equation (1). The manipulation is carried out in ambient conditions, at $20^{\circ} \mathrm{C}$ and a relative humidity of $48 \%$.

\section{A. Haptic Feedback of Nanoscale Interactions}

The haptic feedback is designed to return to the user the nanoscale interactions of the pick-and-place operations as faithfully as possible. It is synthesized from force responses of Tip I and Tip II. As detailed in [17], adhesion forces $F_{a o}$ between the sphere and the substrate can be estimated as:

$$
F_{a o}=F_{I}+F_{I I}
$$

where $F_{I}$ (resp. $F_{I I}$ ) is the force applied to the cantilever I (resp. II). Hence, the haptic force rendered to the user is computed as:

$$
F_{h z}=\alpha_{f}\left[\left(F_{I}-F_{I_{0}}\right)+\left(F_{I I}-F_{I I_{0}}\right)\right]
$$

where $F_{0}=F_{I_{0}}+F_{I I_{0}}$ is the force measured when the tips are holding the sphere before lift-off and it is naturally proportional to the grasping force applied by the tips to the object. Removing this offset allows the user to discard the grasping force which is not useful for pick-and-place. Moreover, in the case where the grasped object is lost hazardously during the lift-off, the measured forces $F_{I}$ and $F_{I I}$ will fall back to zero and Eq. (5) will give a negative value, pulling back the probes to the substrate. $\alpha_{f}$ is a force amplification factor used to scale the measured forces and the haptic force sent to the user. the nominal value used here is $\alpha_{f}=2.0 \cdot 10^{6}$. This coefficient is set considering the magnitude of nanoscale interactions that should be felt by the user (in particular the pull-off force). Detailed discussion on the definition of this parameter can be found in [15].

Fig. 7 represents the haptic feedback during a pick-and-place operation of a $5 \mu \mathrm{m}$ sphere from a glass substrate and in the insert the forces measured from probes.

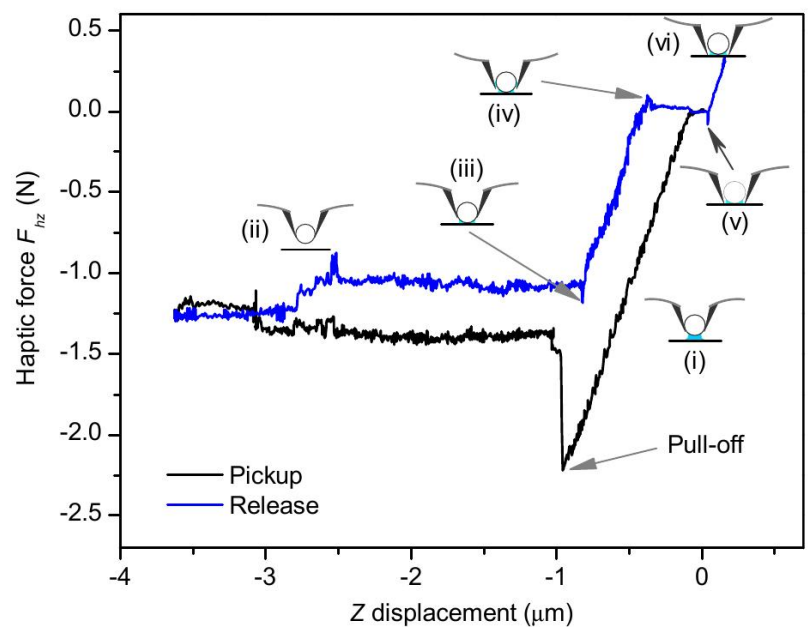

Fig. 7. Synthesized normal force responses from both microcantilevers during the pick-and-place manipulation of a microsphere: (i) pick-up; (ii) The microsphere is detached from substrate after the pull-off; (iii) The microsphere snaps in the substrate; (iv) gripper/microsphere pull-off; (v) The gripper snaps in the substrate; (vi) Manipulation is ended with slight bending of the microcantilevers.

The curve's starting point is the contact state between the microsphere and the substrate. As the nanostage moves down (hence the object held by the tips is lifted), probes are bent down measuring negative forces (inset i). During the pickup, when the nanostage position reaches around $-900 \mathrm{~nm}$, the microsphere pulls off the substrate with a minimum force of $-1125 \mathrm{nN}$ overcoming the adhesion. Note that after the pull-off, the measured force falls to $-550 \mathrm{nN}$, and not to the pre-pick-up null value (inset ii). Actually, as the tip/object contact points are in the lower hemisphere, during the liftoff the object slides slightly down, increasing the grasping

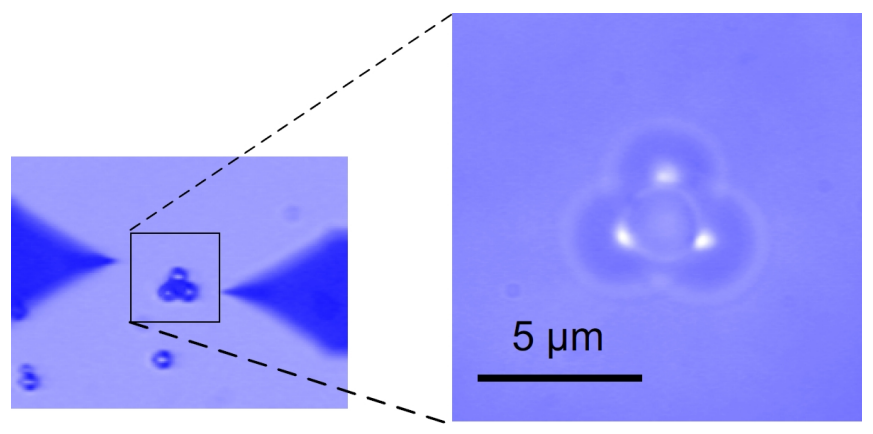

Fig. 8. Teleoperated 3-D microassembly results in a micropyramid. The photos are captured under magnification of $20 \times$ and $100 \times$ respectively. 
force. During the transport phase, a change on the force can be noted. This is again due to the sliding of the sphere in the gripper. This hypothesis is backed-up by the approx. $0.2 \mu \mathrm{m}$ difference seen between pick-up and touch down positions along $z$ axis.

During the release operation the microsphere snaps-in the substrate (inset iii). As the object is pushed to the substrate, between (iii) and (iv) the contact force compensates the grasping force, until the tips pull off the sphere (inset iv) and slide down from the object to the substrate (inset v). At this point, it is sufficient to move apart both tips along $y$ direction to release the sphere from the gripper and achieve the operation. Note that as the contact area at object/tip interface is much smaller than at the object/substrate interface due to the sharp tips used, the problem of the object adhering to a probe is limited.

\section{B. Construction of a Two-layer Pyramid}

In order to validate 3-D manipulation capabilities of the haptic system, four nylon microspheres with diameter of 4$6 \mu \mathrm{m}$ were used to build a two layer pyramid microstructure [18]. Microspheres were deposited on a freshly cleaned glass substrate. An area of interest for the experiments was selected under an optical microscope with $20 \times$ optical magnification. The 3D micropyramid is built using the teleoperated system described in previous sections. The result of this assembly is depicted in Fig. 8.

\section{CONCLUSiON}

A teleoperated 3D microassembly task has been successfully performed thanks to the use of haptic feedback. Although a complex tool composed of two cantilevers as a dual-tip gripper has to be used to perform accurate grasping and pick-and-place at this scale, users unfamiliar with the setup were able to carry out the task as haptic feedback ensures a high intuitiveness of the setup. The use of the cantilever in both static and dynamic mode enabled a complex manipulation using an AFM tool. A two layer pyramid is built from four $\varnothing 5 \pm 1 \mu \mathrm{m}$ spheres in ambient conditions for experimental validation of the dexterity and usability of the overall setup.

The methodology presented in this paper can be applied directly to other type of objects, such as carbon nanotubes or nanowires, except for $y$ axis virtual guides which are specific to spherical objects but can be further extended to nonspherical objects by taking the object geometry in account for in-line generation.

\section{ACKNOWLEDGMENTS}

This work was supported by the ANR (French Agency of Research) through the PACMAN Project, and the NANOROL Project under Grant No. PSIROB07-184846.

\section{REFERENCES}

[1] R. Fearing, "Survey of sticking effects for micro parts handling," in Proceedings of the IEEE/RSJ International Conference on Intelligent Robots and Systems, vol. 2, 1995, pp. 212-217.
[2] A. Menciassi, A. Eisinberg, I. Izzo, and P. Dario, "From "macro" to "micro" manipulation: models and experiments," IEEE/ASME Transactions on Mechatronics, vol. 9, no. 2, pp. 311 -320, 2004.

[3] I. Bukusoglu, C. Basdogan, A. Kiraz, and A. Kurt, "Haptic manipulation of microspheres using optical tweezers under the guidance of artificial force fields," Presence: Teleoperators and Virtual Environments, vol. 17, no. 4, pp. 344-364, 2008.

[4] M. Guthold, M. Falvo, W. Matthews, S. Paulson, S. Washburn, D. Erie, R. Superfine, F. Brooks Jr., and R. Taylor II, "Controlled manipulation of molecular samples with the nanomanipulator," in Proceedings of the International Conference on Advanced Intelligent Mechatronics, 1999, pp. 3-8.

[5] A. Ferreira and C. Mavroidis, "Virtual reality and haptics for nanorobotics," IEEE Robotics and Automation Magazine, vol. 13, no. 3, pp. 78-92, 2006.

[6] R. Hollis, S. Salcudean, and D. Abraham, "Toward a tele-nanorobotic manipulation system with atomic scale force feedback and motion resolution," in Proceedings of the IEEE conference on Micro Electro Mechanical Systems, 1990, pp. 115-119.

[7] S. Marliere, D. Urma, J. Florens, and F. Marchi, "Multi-sensorial interaction with a nano-scale phenomenon: The force curve," in Proceedings of the Eurohaptics, 2004, pp. 246-252.

[8] G. Li, N. Xi, H. Chen, P. Craig, and P. Mathew, "Videolized atomic force microscopy for interactive nanomanipulation and nanoassembly," IEEE Transactions on Nanotechnology, vol. 4, no. 5, pp. 605-615, 2005.

[9] M. Guthold, M. Falvo, W. Matthews, S. Paulson, J. Mullin, S. Lord, D. Erie, S. Washburn, R. Superfine, F. B. Jr., and R. T. II, "Investigation and modification of molecular structures with the nanomanipulator," Journal of Molecular Graphics and Modelling, vol. 17, pp. 187 - 197, 1999.

[10] M. Sitti and H. Hashimoto, "Teleoperated touch feedback from the surfaces at the nanoscale: modeling and experiments," IEEE/ASME Transactions on Mechatronics, vol. 8, no. 2, pp. 287-298, 2003.

[11] G. Li, N. Xi, M. Yu, and W.-K. Fung, "Development of augmented reality system for afm-based nanomanipulation," IEEE/ASME Transactions on Mechatronics, vol. 9, no. 2, pp. 358-365, 2004.

[12] W. Vogl, B. Ma, and M. Sitti, "Augmented reality user interface for an atomic force microscope-based nanorobotic system," IEEE Transactions on Nanotechnology, vol. 5, no. 4, pp. 397-406, 2006.

[13] C. D. Onal and M. Sitti, "Teleoperated 3-D force feedback from the nanoscale with an atomic force microscope," IEEE Transactions on Nanotechnology, vol. 9, no. 1, pp. 46-54, 2010.

[14] M. Sitti, "Survey of nanomanipulation systems," in Proceedings of the IEEE Conference on Nanotechnology, 2001, pp. 75 -80.

[15] A. Bolopion, B. Cagneau, S. Haliyo, and S. Régnier, "Analysis of stability and transparency for nanoscale force feedback in bilateral coupling," Journal of Micro - Nano Mechatronics, no. 4, pp. 145$158,2009$.

[16] A. Bolopion, B. Cagneau, and S. Régnier, "2D micro teleoperation with force feedback," in Proceedings of the IEEE International Conference on Intelligent Robots and Systems, 2009, pp. 3265-3570.

[17] H. Xie and S. Régnier, "Three-dimensional automated micromanipulation using a nanotip gripper with multi-feedback," Journal of Micromechanics and Microengineering, vol. 19, no. 7, p. 075009 (9pp), 2009.

[18] H. Miyazaki and T. Sato, "Pick and place shape forming of threedimensional micro structures from fine particles," in Proceedings of the IEEE International Conference on Robotics and Automation, vol. 3, 1996, pp. 2535-2540. 